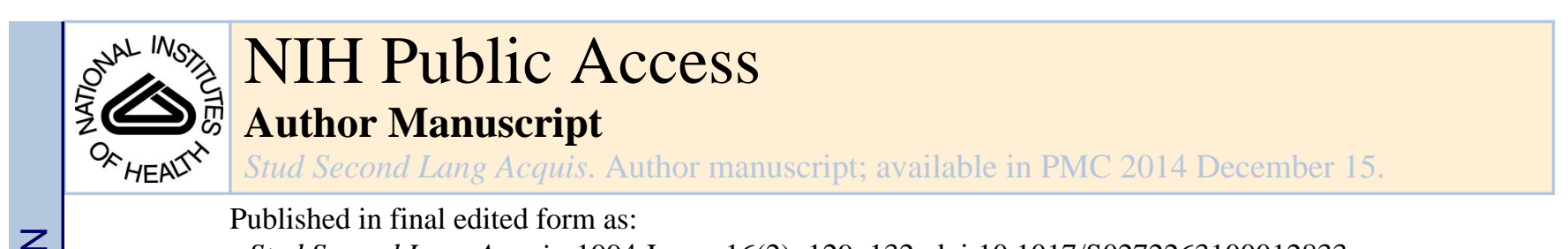

Published in final edited form as:

Stud Second Lang Acquis. 1994 June ; 16(2): 129-132. doi:10.1017/S0272263100012833.

\title{
COGNITIVE FOUNDATIONS OF SECOND LANGUAGE ACQUISITION
}

\author{
Russell S. Tomlin and \\ University of Oregon \\ Morton A. Gernsbacher \\ University of Wisconsin-Madison
}

\section{Introduction}

The growth of cognitive science as a field, or at least as a descriptive term, indicates an emerging scientific consensus that many fundamental human capacities require collaborative and interdisciplinary research to make further fundamental headway. Language, as central to our essential humanity as anything is, represents one area in which massive amounts of interdisciplinary research is underway at virtually every research institution in the world.

It strikes us, then, how comparatively little interdisciplinary research there is within cognitive science about second language acquisition (SLA). There is, of course, a great deal of research within SLA itself that draws upon research in cognition and that extends those ideas in important and interesting ways into both SLA and second language instruction (SLI). Yet SLA has never really taken a particularly prominent place within cognitive science overall. Even a brief glance at recent proceedings of the Cognitive Science Society reveals comparatively little effort directed at SLA. The irony is doubled when one considers that SLA has as a discipline properly taken pride in its multidisciplinary roots. It combines ideas and research strategies from linguistics, sociology, anthropology, education, psychology, and even biology as it examines the myriad difficulties of describing and explaining how individuals fail and succeed in learning additional languages.

In the winter of 1992, a symposium was held at the University of Oregon in Eugene on the topic of cognition, SLA, and SLI. The symposium was sponsored principally by a grant from the Keck Foundation, which supported a general program in cognition and instruction in the University of Oregon Institute for Cognitive and Decision Sciences. ${ }^{1}$ The purpose of this meeting was to assemble researchers in the three related areas of cognition, SLA, and SLI to try to identify and define research questions that cut across the three areas. Approximately 30 participants from around the world and representing varied theoretical approaches to SLA worked together for 3 days looking for points of contact among their various perspectives on SLA, SLI, and cognition. ${ }^{2}$ The present issue is one product of that event.

(c) 1994 Cambridge University Press

${ }^{1}$ The symposium was also supported by the Yamada Language Center and the American English Institute, University of Oregon.

2 And while there were many notable participants in this venture, there were at least as many notable voices that were not heard. 
The contributions in this issue span a continuum from those whose bases are principally within SLA but tied to issues of cognition to those that are largely grounded in the more general cognitive literature but tied to issues of importance in SLA. Andersen and Shirai examine the relationship between child language acquisition and SLA of tense-aspect distinctions. They argue that there are several fundamental cognitive principles underlying the pattern of acquisition seen in the data: the Relevance Principle, the One to One Principle, and the Principle of Congruence. They argue further that these principles are not peculiar to acquisition per se but are in fact principles that govern ordinary native speaker discourse interaction.

Bialystok presents a distillation of key ideas from her long-standing research program on analysis and control in SLA. Both analysis and control are fundamentally cognitive ideas about the language learning capacity, and Bialystok considers how these ideas address five central problems of SLA: the similarity between first and second language acquisition, the starting point for SLA, consciousness, variability, and instruction.

Birdsong introduces a new research arena for SLA. A number of years ago, Tversky and Kahnemann (1974) examined how humans routinely misjudge the frequency of events they encounter. These misjudgments stem from the employment of general heuristics for estimating how frequently events occur. Although commonly successful, these heuristics nonetheless routinely lead to mistaken and biased judgments; for example, estimates of the product of $6 \times 5 \times 4 \times 3 \times 2$ tend to be higher than those of the mathematically identical expression $2 \times 3 \times 4 \times 5 \times 6$ because of the anchoring point at which the estimates begin. If such biases operate in many other domains where hypotheses about specific phenomena are formed, then it is intriguing to consider how those same heuristics and biases may affect formation of interlanguage hypotheses.

Tomlin and Villa present a detailed discussion of attention and SLA. While it has long been recognized that attention plays a central role in acquisition, our views of attention have often been somewhat naive, combining folk ideas of attention with more technical ones. The authors provide a detailed review of the attention literature in cognitive psychology and argue that a more detailed model of the attentional system may help SLA formulate a finer grained model of the role attention plays in the online and real-time process of acquisition.

Carr and Curran present a detailed examination of implicit learning. A problem of considerable interest in SLA has been whether SLA can occur without conscious or aware engagement of input by the learner. The issue is particularly difficult to examine because of theoretical difficulties in specifying what implicit or nonconscious learning means and because of tremendous methodological problems in investigating such issues. This article reviews both the theoretical issues of defining clearly what implicit learning is and the methodological issues of investigating implicit learning carefully in limited domains.

Schumann examines the neurobiological basis of the so-called affective filter in SLA. In doing so, he raises two issues of importance to SLA. First, he challenges whether research into affective constraints on SLA should take the secondary place in the field it has in recent years. Second, he challenges simplistic views of cognition, as well, by arguing that cognitive 
theories must recognize their ultimate connection to the architecture in which they are situated. ${ }^{3}$ In a sense, he argues from neurobiological grounds that we cannot afford to treat affective issues as secondary to rational ones in formulating SLA theory.

Despite what I hope readers will find worthwhile papers, this issue is missing work that our ideal issue would have included. There are no articles that address directly issues relating cognition and SLA to instruction. The Editor and the Editorial Board of SSLA along with the volume editors believed it to be important to include research that identified issues in instruction that might be addressed by interaction of cognitive science and SLA. Such issues did form part of the symposium, and we did attempt to include this coverage in the present effort. However, circumstances in the end did not permit us to include such a paper and this remains a gap in what we have offered in this thematic issue. Perhaps another will find the bridge between cognition and instruction easier to construct, setting a new theme for a future SSLA special issue.

One final observation is in order. At first glance the contribution this issue makes will once again seem mostly to tell us in SLA that we would benefit from a more careful and detailed extension of ideas and methods from cognitive science into SLA. Carr and Curran present elaborate argumentation on issues of implicit learning. Tomlin and Villa argue for an increasingly complex model of the role of attention in SLA. Birdsong links SLA to cognitive biases tied to decision making in general. Schumann examines the neurological basis for the affective filter. Such extensions may well benefit our general research mission, and we hope very much that readers will find these contributions useful. But these same papers also make a case for incorporating the study of SLA more directly into the research agenda of cognitive science. SLA is a process in which matters about language that are ordinarily confounded may be separated. Child language acquisition faces unavoidable confounding of language acquisition with other aspects of child cognitive and biological development. The variability inherent in interlanguage grammars poses a real challenge to tidy theoretical conceptions of the nature of linguistic representations. The complication of examining the interplay between SLA and SLI may help clarify enduring problems about the interplay between social interaction (teaching) and cognitive change (learning). In these areas, along with many others, SLA has as much to offer cognitive science as cognitive science does SLA. Put more simply, SLA has always been a part of cognitive science, and we, researchers in this vital area, very much cognitive scientists.

\section{References}

Tversky A, Kahnemann D. Judgment under uncertainty: Heuristics and biases. Science. 1974; 185:1124-1131. [PubMed: 17835457]

\footnotetext{
${ }^{3}$ It is also a pleasure to note parenthetically that Schumann has done an outstanding job of dealing with the neuroscience literature. One pervasive fear for such articles is that they may overstate or miss critical details that experts in the other discipline would not. But this article, reviewed by one leading neuroscientist working specifically in the area of emotion and the amygdala, meets both the tough standards of SLA and those of neuroscience publication.
} 